\title{
THE EFFECT OF TIME CONSTRAINTS ON OLDER AND YOUNGER DRIVER DECISIONS TO TURN AT INTERSECTIONS USING A MODIFIED CHANGE BLINDNESS PARADIGM
}

\author{
J.K. Caird, C.J. Edwards and J. Creaser \\ Cognitive Ergonomics Research Laboratory \\ University of Calgary, Canada; \\ C.T. Scialfa and W.J. Horrey \\ Beckman Institute \\ University of Illinois, U.S.
}

Summary: After age 75, the risk of intersection accident involvement for older drivers increases for most intersection maneuvers. Failure to yield right-of-way and violation of traffic controls are common citations. Previous research has argued that age-related declines in attentional breadth and switching can discriminate between those who are and are not more likely to be in an accident.

The present study examined the effect of time constraints on older and younger driver intersection decisions. It was expected that less time to decide, process and act upon intersection decisions would adversely affect older drivers more so than younger drivers. The change blindness or flicker paradigm was modified to address these questions (see, e.g., Rensink, et al., 1997). Typically, an image (A) is alternated with a modified image (A') each for a short duration (250 ms) with a blank field or mask ( $80 \mathrm{~ms}$ ) between A and A'. In the present study, a focus screen (or prime) that indicated the expected direction of travel (i.e., left, right, or straight) was added prior to the alternation of images. Forty-eight images were selected from 2500 intersection approaches in Calgary, Winnipeg and Montreal that were filmed using a digital camera during the day. Photoshop was used to create the A' images by manipulating a variety of elements including pedestrians, vehicles, signs, and signals. The goal of the participant was to decide if the indicated direction of travel in the pair of intersection images was safe (press accelerator) or not (press brake).

Sixteen younger $(\underline{\mathrm{M}}=22.3)$ and 16 older drivers $(\underline{\mathrm{M}}=73.6)$ drivers were screened for visual acuity, contrast sensitivity, and medication use. Length of image alternation was the primary independent variable (4 and $8 \mathrm{~s}$ ). As expected, older drivers were more likely to miss intersection changes at shorter exposure durations than younger drivers. A number of qualitative probes were also collected that clearly illustrate the contextual demands of intersections on attention. The implications of the results for intersection design and older driver selective attention are discussed. 